\title{
Supplemental growth hormone in healthy adults: the endocrinologist's responsibility
}

\author{
Shlomo Melmed
}

In the US, growth hormone (GH) administration has been approved by the FDA for adults with proven acquired pituitary deficiencies, including those arising from hypothalamic-pituitary masses, surgery, irradiation or infiltrative processes. Adults with childhood-onset $\mathrm{GH}$ deficiency also benefit from FDA-approved $\mathrm{GH}$ replacement. $\mathrm{GH}$ is also approved for treating AIDS-related muscle wasting in adults. In patients who fulfill the biochemical and clinical criteria for adult GH deficiency, physiologic replacement improves quality of life, enhances lean body mass, improves cardiovascular function and could strengthen bone (Cummings DE and Merriam GR Ann Rev Med 2003, 54: 513-533). Prospective efficacy and safety surveillance studies are currently underway.

Side-effects of adult $\mathrm{GH}$ administration are reported in up to $30 \%$ of patients and are usually dose-dependent. They include myalgias, carpal tunnel syndrome, edema, elevated blood pressure, left ventricular remodeling, hyperglycemia and clinical diabetes. No increased cancer occurrence has been observed in surveillance studies of $\mathrm{GH}$ replacement in pituitary-deficient adults; however, accurate information for the impact of uncontrolled use of $\mathrm{GH}$ on cancer and cardiovascular function is not available.

Positive effects of $\mathrm{GH}$ on nitrogen retention, enhanced lean body mass and loss of fat mass have resulted in widespread nonapproved use of $\mathrm{GH}$ to reverse, halt, or decelerate the frailty of advancing age or to enhance athletic performance. Approximately 30,000 individuals are reported to receive GH for 'anti-aging' therapy annually but there are no compelling clinical trials indicating safety and benefits of $\mathrm{GH}$ administration to the elderly versus a disciplined diet and exercise training regimen; indeed, GH concentrations normally decline with aging and several animal models point to a protective effect of age-related low GH levels resulting in increased lifespan (Sonntag WE et al. Endocrinology 2005, 146: 2920-2932).

\section{It is highly \\ likely that \\ a large \\ proportion of \\ GH taken by \\ adults is in fact \\ both clinically \\ and legally \\ inappropriate}

$S$ Melmed is Professor and Director of the Research Institute, Cedars-Sinai Medical Center, Los Angeles, CA, USA.

\section{Competing interests} The author declared competing interests; go to the article online for details.

www.nature.com/clinicalpractice doi:10.1038/ncpendmet0138
Whether physiologic lower GH levels are in fact permissive for a longer lifespan, protect cardiac function and lower cancer incidence requires rigorous controlled study. Thus antiaging indications for $\mathrm{GH}$ are presently considered inappropriate, unsafe, illegal and not based upon evidence for a positive sustained impact on muscle function (Lange KHW et al. J Clin Endocrinol Metab 2002, 87: 513-523; Berggren A et al. J Clin Endocrinol Metab 2005, 90: 3268-3273).

It has been estimated that 100,000 individuals in the US obtain GH without a prescription every year. In addition 160,000 new and refill GH prescriptions for adults are completed by retail and mail service pharmacies (Perls TH et al. JAMA 2005, 294: 2086-2090), but it is difficult to assess accurately how many are truly $\mathrm{GH}$ deficient with documented pituitary dysfunction. It is highly likely that a large proportion of GH taken by adults is in fact both clinically and legally inappropriate. Yet, off-label distribution of $\mathrm{GH}$ carries significant financial penalties and can lead to imprisonment (Perls TH et al. JAMA 2005, 294: 2086-2090).

In today's era of increasing public awareness of healthcare quality outcomes and cost containment for healthcare, it behooves responsible endocrinologists to maintain rigorous scientific, clinical and legal criteria for prescribing GH. Primum non nocere remains our responsible approach to prescribing of all medication. Without sufficient scientifically based evidence for anti-aging effects of $\mathrm{GH}$, our professional societal responsibility is to adhere to regulatory guidelines appropriately restricting expensive ( US $\$ 8,000$ or more per annum) adult GH therapy to bona fide replacement needs.

As endocrinologists, our informed response to queries from patients and referring physicians should be tempered with caution that neither the safety nor the benefits of $\mathrm{GH}$ administration to pituitary-replete adults have been defined for this very costly hormonal intervention. 